\title{
DIFFERENTIAL EQUATIONS OF THE MATRIX ELEMENTS OF THE DEGENERATE SERIES
}

\author{
BY ROBERT HERMANN
}

Communicated by R. S. Palais, June 12, 1967

1. Introduction. Let $G$ be a noncompact, semisimple Lie group, and let $U(G)$ be the universal enveloping algebra of its Lie algebra. [For notations used here, see [3].] Consider an irreducible representation $\rho$ of $G$ on a Hilbert space $H$. Let $I(G)$ be the central elements in $U(G)$, i.e., the Casimir operators of the Lie algebra. Then, for $\Delta \in I(G), \rho(\Delta)$ is a multiple of the identity in $H$, which implies that the matrix elements of the representation, considered as functions on $G$, satisfy certain invariant differential equations, whose nonconstant terms are just the differential operators on $G$ resulting from considering the elements of $I(G)$ as differential operators on $G$.

Following a suggestion of $A$. Koranyi, we now ask the following question. Suppose $\Delta$ is an element of $U(G)$ that is not in the center. Are there representations $\rho$ which have the property that $\rho(\Delta)$ is a multiple of the identity also, hence that the matrix elements of $\rho$, considered as functions on $G$ satisfy additional differential equations derived from $\Delta$ ? A remark of this type might be useful in interpreting the role that the various types of representations play in the Plancherel formula for functions on the group, and in characterizing by differential equations the classes of harmonic functions on $G / K$ ( $K=$ maximal compact subgroup) arising from various Poisson integral formulae.

In this note, we indicate how to find such $\Delta$ 's for certain induced representations of $G$. Roughly, we show that the higher the degree of degeneracy of the representation, the more likely one is to find such operators. We use a differential geometric method already given in [2] and [3] to prove the vanishing of certain differential operators.

2. Conditions for the vanishing of differential operators. Consider a space with variables $\left(x_{i}\right)=x$ (choose the range of indices $1 \leqq i, j$, $\cdots, \leqq n)$. Let $\Delta$ and $X$ be linear differential operators on this space, of order $r$ and 1 respectively. Then

$$
\Delta=\sum A_{i_{1}} \cdots i_{i_{r}} \frac{\partial^{r}}{\partial x_{i_{1}} \cdots \partial x_{i_{r}}}+(\cdots)
$$

where $A_{i_{1}} \cdots i_{i_{r}}(x)$ are functions on this space, depending symmetrically on the indices. [The terms $(\cdots)$ are of lower order.] 
Suppose that $X$ is of the form:

$$
\sum \lambda_{i} x_{i} \frac{\partial}{\partial x_{i}}+f(x)=X^{\prime}+f(x)
$$

where $\left(\lambda_{i}\right)$ are constants, and $f(x)$ a function. Suppose also a relation of the following form between $X$ and $\Delta$ :

$$
[X, \Delta]=\lambda \Delta
$$

with real $\lambda$, where [ , ] is the commutator of two differential operators.

Now

$$
\begin{gathered}
{\left[X, \frac{\partial}{\partial x_{k}}\right]=-\lambda_{k} \frac{\partial}{\partial x_{k}}+(\cdots)} \\
{[X, \Delta]=\sum X^{\prime}\left(\left(A_{i_{1} \cdots i_{r}}\right)-A_{i_{1} \cdots i_{r}}\left(\lambda_{i_{1}}+\cdots+\lambda_{i_{r}}\right)\right) \frac{\partial^{r}}{\partial x_{i_{1}} \cdots \partial x_{i_{r}}}+\cdots}
\end{gathered}
$$

Comparing this with (2.2), we have

$$
X^{\prime}\left(A_{i_{1} \ldots i_{r}}\right)=A_{i_{1} \cdots i_{r}}\left(\lambda+\lambda_{i_{1}}+\cdots+\lambda_{i_{r}}\right) .
$$

Taking the value of both sides at $x=0$ gives

$$
A_{i_{1} \ldots i_{r}}(0)\left(\lambda+\lambda_{i_{1}}+\cdots+\lambda_{i_{r}}\right)=0 .
$$

Then we have proved

TheOREM 2.1. Suppose that

$$
\lambda+\lambda_{i_{1}}+\cdots+\lambda_{i_{r}} \neq 0 \text { for } 1 \leqq i, i_{1} i_{2} \cdots i_{r} \leqq n .
$$

Then, $A_{i_{1} \cdots i_{r}}(0)=0$, i.e., the highest order terms of $\Delta$ vanish at $x=0$.

3. Induced representations. Let $G$ be a connected, semisimple, noncompact Lie group with a finite center. Let $K$ be a maximal compact subgroup, $G=K+P$ the Cartan decomposition, i.e.,

$$
[K, K] \subset K ; \quad[K, P] \subset P ; \quad[P, P] \subset K .
$$

Let $X$ be an element of $P$. Then, $\operatorname{Ad} X$ has real eigenvalues. Let $\mathbf{C}(X)$ be the centralizer of $X$ in $G, N^{+}(X)$ and $N^{-}(X)$ the (nilpotent) subalgebras formed by the eigenvectors of $\operatorname{Ad} X$ with positive and negative eigenvalues. Then

$$
G=C(X) \oplus N^{+}(X) .
$$

Let $C(X), N^{-}(X), N^{+}(X)$ be the connected subgroups of $G$ generated by the corresponding subalgebras of $G$. Let $H$ be the subalgebra 
$\mathrm{C}(X)+\mathrm{N}^{-}(X)$, and $H$ the corresponding subgroup. Then $H$ is one of the boundary subgroups and $K$ acts transitively on $G / H$.

Let $p_{0}$ be the identity coset of $G / H$. Consider the orbit $N^{+} p_{0}$. It is known that it is an open set in $G / H$, and its complement is a union of a finite number of submanifolds of lower dimension. Let $Y_{1}, \ldots$, $Y_{n}$ be a basis of $N^{+}$consisting of eigenvectors of Ad $X$. Let $x_{1}, \cdots, x_{n}$ be "canonical co-ordinates" on the orbit $N^{+} p_{0}$ with respect to this basis, i.e.,

$$
x_{i}\left(\operatorname{Exp}\left(t_{1} Y_{1}\right) \cdots \operatorname{Exp}\left(t_{n} Y_{n}\right) \cdot p_{0}\right)=t_{i}, \quad 1 \leqq i \leqq n .
$$

Then, the action of $\exp t X$ on $G / H$ is linear in these co-ordinates. Further, the volume element $d x=d x_{1} \cdots d x_{n}$ is invariant under the action of $N^{+}$. Use $d x$ to construct an inner product on the space $F(G / H)$ of complex valued functions on $G / H$.

$$
(f, f)=\int f(p) d x \text { for } f \in F(G / H) .
$$

( $p$ denotes a typical point of $G / H$.) Construct a representation $\rho$ of $G$ on $F(G / H)$ in the standard way:

$$
\rho(g)(f)(p)=m(g, p) f\left(g^{-1} p\right)
$$

for $g \in G, p \in G / H$. [ $m(g, p)$ is a multiplier system for the action of $G$ on $G / H$.]

Bruhat [1] has given the most extensive treatment of this general situation and we can refer there for a full discussion of the relation to the work of Bargmann, Gel'fand and Neumark, and Mackey.

Our constructions imply that $\rho(X)$ is of the form (2.1) with eigenvalues $\lambda_{1}, \cdots, \lambda_{n}$ just the eigenvalues of $\operatorname{Ad} X$ on $Y_{1}, \cdots, Y_{n}$. Suppose $\Delta: F(G / H) \rightarrow F(G / H)$ is a differential operator satisfying (2.2). Then, under conditions on $\lambda, \lambda_{1}, \cdots, \lambda_{n}$ and $r$ made precise in the last section, we may conclude that the highest order terms of $\Delta$ vanish at $p_{0}$. We now present more conditions which, if satisfied, imply that $\Delta$ is a zeroth order operator.

Let us forget about the conditions of $\$ 2$ for the moment. Suppose $\Delta_{1}, \cdots, \Delta_{m}$ are differential operators: $F(G / H) \rightarrow F(G / H)$ which transform among themselves under $\rho(G)$, i.e.,

$$
\rho(g) \Delta_{u} \rho\left(g^{-1}\right)=a_{u v}(g) \Delta_{v}
$$

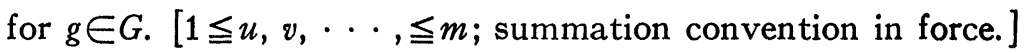

Then, also 


$$
\left[\rho(X), \Delta_{u}\right]=a_{u v}(X) \Delta_{v}
$$

$g \rightarrow\left(a_{u v}(g)\right)$ and $X \rightarrow\left(a_{u v}(X)\right)$ are matrix representations of $G$ and $G$. Suppose that we knew that the highest terms of all the operators $\Delta_{1}, \cdots, \Delta_{m}$ vanished at $p_{0}$. We will now show that we can conclude that the highest order terms vanish identically on $G / H$.

Lemma 3.1. Suppose $\Delta_{1}^{\prime}, \cdots, \Delta_{m}^{\prime}$ are the highest order differential operators occurring for the operators $\Delta_{1}, \cdots, \Delta_{m}$, i.e., $\Delta_{u}=\Delta_{u}^{\prime}+$ (operators of lower order), $1 \leqq u \leqq m$. Suppose each $\Delta_{u}^{\prime}$ is an operator of order $>0$. Let $\rho^{\prime}(g)$ be the transformation $\psi(p) \rightarrow \psi\left(g^{-1} p\right)$ on $F(G / H)$. Then,

$$
\rho(g) \Delta_{u}^{\prime} \rho\left(g^{-1}\right)=\rho^{\prime}(g) \Delta_{u} \rho^{\prime}\left(g^{-1}\right)+(\cdots)=a_{u v}(g) \Delta_{v}^{\prime}+(\cdots)
$$

for $g \in G$.

The proof should be obvious. For the transformation law $\psi(p)$ $\rightarrow m(g, p) \psi\left(g^{-1} p\right)=\rho(g)(\psi)(p)$, the multipliers will have no effect on the terms of highest order, whence (3.2).

A similar argument proves the following:

Lemma 3.2. Suppose the representation $g \rightarrow\left(a_{u v}(g)\right)$ is irreducible. Then, all the operators $\Delta_{1}^{\prime}, \cdots, \Delta_{m}^{\prime}$ have the same degree.

LemMa 3.3. Suppose $\Delta_{1}^{\prime}, \cdots, \Delta_{m}^{\prime}$ all vanish at one point of $G / H$. Then they vanish identically on $G / H$.

We can now combine these remarks with those of $\S 2$, to obtain

THEOREM 3.1. Suppose $\Delta_{1}, \cdots, \Delta_{m}$ are differential operators $F(G / H) \rightarrow F(G / H)$ of order $\leqq r$, with $r>0$, transforming among themselves under $\rho(G)$ by a finite dimensional irreducible representation. Suppose $\Delta_{1}, \cdots, \Delta_{m}$ are, further, chosen as eigenvectors of $\rho(X)$, i.e.,

$$
\left[\rho(X), \Delta_{u}\right]=\alpha_{u} \Delta_{u} .
$$

Suppose $\rho^{\prime}(X)=\sum_{i} \lambda_{i} x_{i}\left(\partial / \partial x_{i}\right)$, and

$$
\alpha_{u}+\lambda_{i_{1}}+\cdots+\lambda_{i_{r}} \neq 0 \text { for } 1 \leqq i, i_{1}, \cdots, i_{r} \leqq n, 1 \leqq u \leqq m .
$$

Then, the terms of order $r$ vanish identically on $G / H$. In particular, if

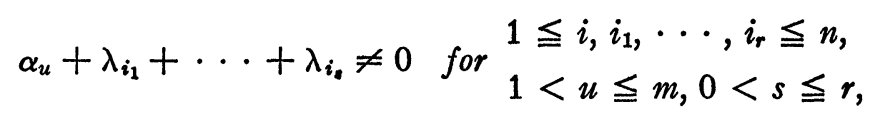

then $\Delta_{1}, \cdots, \Delta_{m}$ are zeroth order differential operators on $G / H$.

Now, the action of $U(G)$ on $F(G / H)$ gives us a supply of differential operators which transform under $\rho(G)$ by finite dimensional irre- 
ducible representations. It is known from Kostant's work [4] that $U(G)$, considered as an $I(G)$ module, is free, and each irreducible representation of $G$ occurs (under $\operatorname{Ad} G$ ) at most a finite number of times. [This number is equal to the number of invariants of a Cartan subalgebra of $G$ in the representation space.]

This argument is easiest to apply if the operators are invariant under $\rho(G)$, i.e., if they come from $I(G)$, for then (3.3) is satisfied for all $r>0$ [since $\alpha_{u}=0$, and $\lambda_{i}>0$ for $1 \leqq i \leqq n$ ]. If, however, the $\Delta_{1}, \cdots, \Delta_{m}$ transform by a nontrivial representation of $G$, obviously the analysis of the condition (3.4) involves a detailed study of the roots of the symmetric space $G / K$, how the element $X$ sits in a Weyl chamber of $P$, and how the weights of the representation restrict to $X$. Rather than go in to these details here, we will consider just one case:

Theorem 3.2. Suppose that Ad $X$ acting in $N^{+}$has a single eigenvalue, i.e., $\lambda_{1}=\cdots=\lambda_{n}$, i.e., $N^{+}$is Abelian and $H$ is a maximal subalgebra of $G$. Suppose also that the $\Delta_{1}, \cdots, \Delta_{m}$ transform among themselves by the adjoint representation. Then, $\Delta_{1}, \cdots, \Delta_{m}$, as differential operators on $F(G / H)$, are of degree at most one.

For the proof, notice that $m=n$ and $\alpha_{u}$ can be either $0, \lambda_{i}$, or $-\lambda_{i}$, which is only compatible if $r=1$.

Now, the operators $\rho(Y)$, for $Y \in G$, are differential operators on $F(G / H)$ of degree one. If there is but one set of first order operators on $F(G / H)$ transforming in this way (a question decidable using the Frobenius reciprocity theorem [3]), then some linear combination of the $\Delta$ 's and the $\rho(Y)$ 's must be zeroth order differential operators, if the conditions of Theorem 3.2 are satisfied.

\section{REFERENCES}

1. F. Bruhat, Sur les representations induites des groupes de Lie, Bull. Soc. Math. France 84 (1956), 97-205.

2. R. Hermann, Geometric aspects of potential theory in symmetric spaces, Ann. of Math., Part I in 148 (1962), 349-366; Part II in 151 (1963), 143-149; Part III in 152 (1964), 384-394.

3. - Lie groups for physicists, Benjamin, New York, 1965.

4. B. Kostant, Lie group representations on polynomial rings, Amer. J. Math. 85 (1963), 327-404.

\section{University of California, Santa Cruz and \\ Stanford Linear Accelerator Center}

